\title{
Investigating sluggish diffusion in a concentrated solid solution alloy using ion irradiation with in situ TEM
}

\author{
Matheus A. Tunes ${ }^{\mathrm{a}, 1, *}$, Hoang Le ${ }^{\mathrm{a}}$, Graeme Greaves ${ }^{\mathrm{a}}$, Cláudio G. Schön ${ }^{\mathrm{c}}$, Hongbin \\ Bei $^{\mathrm{b}}$, Yanwen Zhang ${ }^{\mathrm{b}}$, Philip D. Edmondson ${ }^{\mathrm{b}}$, Stephen E. Donnelly ${ }^{\mathrm{a}}$ \\ ${ }^{a}$ School of Computing and Engineering, University of Huddersfield, United Kingdom \\ ${ }^{b}$ Materials Sciences and Technology Division, Oak Ridge National Laboratory, United States of \\ America \\ ${ }^{c}$ Department of Metallurgical and Materials Engineering, Escola Politécnica da Universidade de \\ São Paulo, Brazil.
}

\begin{abstract}
Concentrated solid solution alloys (CSAs) - including high entropy alloys (HEAs) are known for their remarkable mechanical and corrosion resistances with superior tolerance against the deleterious effect of irradiation exposure when compared with pure metals and dilute alloys. To date, however, the mechanisms responsible for such improvements are still unclear and remain a subject of investigation. The present work reports in situ Transmission Electron Microscopy (TEM) study under simultaneous ion irradiation of the face-centred cubic (FCC) FeCrMnNi quaternary HEA, comparing with a non-equiatomic Fe-based alloy, the AISI-348 austenitic stainless steel that has $\mathrm{Cr}, \mathrm{Ni}$ and $\mathrm{Mn}$ as alloying elements. The alloys were irradiated under the same conditions, with $6 \mathrm{keV} \mathrm{He}^{+}$and $134 \mathrm{keV} \mathrm{Xe}^{+}$ions at $298 \mathrm{~K}$ up to $1.7 \times 10^{17}$ ions $\cdot \mathrm{cm}^{-2}$ (4 displacements per atom, dpa) and $2.7 \times 10^{15} \mathrm{ions} \cdot \mathrm{cm}^{-2}$ (4 dpa), respectively. The nucleation of inert gas bubbles was tracked upon post-irradiation extended annealing up to $673 \mathrm{~K}$. He and Xe bubbles were observed to grow at a rate slightly slower in the equiatomic alloy. Trends from the bubble size analyses show that the nucleation and growth of inert gas bubbles are suppressed or delayed in some conditions in the nearly equiatomic alloy.
\end{abstract}

Keywords: High-Entropy Alloys, Irradiation effects, In situ Transmission Electron Microscopy, Diffusion

\footnotetext{
*Corresponding author: Tel. +4401484 471689

Email address: matheus.tunes@unileoben.ac.at (Matheus A. Tunes)

${ }^{1}$ Present address: Chair of Non-ferrous Metallurgy, Montanuniversität Leoben, Franz-JosefStraße 18, A-8700 Leoben, Austria.
} 


\section{Introduction}

Complex concentrated solid solution alloys (CSAs), also known as high-entropy alloys (HEAs), have recently attracted the attention of scientific community due to the scientific potential for high-demanding applications. In opposition to conventional dilute solid solution alloys, CSAs are made with multiple principal elements, two or more, in (near) equiatomic compositions [1]. The maximisation of the configurational entropy in CSAs is believed to be part of the reason to stabilise them against the precipitation/decomposition of complex intermetallic or secondary phases, by lowering their Gibbs free energy of the system [1-4, 4-7]. This has been recently associated with enhancements of several metallurgical properties [8] including enhanced ductility and fracture toughness [9, 10]. For example, an important coreeffect of CSAs is on the nature of atomic diffusion of alloying elements within the random concentrated solid solution [11-19]. Research on point defect mobility in CSAs began with the studies of Tsai et al. [20] who postulated that the diffusion of alloying elements in these new metallic alloys can be considered "sluggish" when compared with reference elemental metals. Despite all these efforts, a more appropriate theoretical-experimental justification for this important core-effect is yet to be addressed $[4,5,21]$.

A prominent field of scientific research on CSAs is their radiation resistance by controlling defect mobility and migration pathways during energetic particle irradiation exposure [22]. Modelling of the radiation resistance of a $\mathrm{ZrNbHf} \mathrm{HEA}$ suggests that the high stresses posed by the atomic size mismatch are responsible for facilitating the process of amorphisation under irradiation when followed by local melting and re-crystallisation promoted by thermal spikes [23]. Other experimental studies have shown that this system - in a form of a nanocrystalline solid solution alloy - was able to retain its matrix phase with no irradiation-induced transformation under exposure to a high-energy electron beam, up to damage levels of 10 displacements-per-atom (dpa) [23-25].

Another CSA, a nanocrystalline $\mathrm{CoCuCrFeNi}[26]$, has been reported to possess impressive irradiation resistance and phase stability up to 40 dpa: at this high damage level, neither secondary phase formation nor grain coarsening were observed under the $\mathrm{MeV}$ electron irradiation. In a recent study of nanocrystalline $\mathrm{CoCuCr}$ FeNi under $3 \mathrm{MeV}$ Ni irradiation to nearly 600 dpa, the FCC phase is observed to be stable with insignificant grain growth (from 15.6 to $25.2 \mathrm{~nm}$ ) [27].

An investigation of the mechanisms of damage production in a NiCoCr CSA using $3 \mathrm{MeV} \mathrm{Au}$ ions, comparing the response with the elemental components under the same conditions, was conducted by Granberg et al. [28]. In their work, defect 
clusters and interstitial-type dislocation loops were observed to form in the CSA for doses as low as $0.1 \mathrm{dpa}$, but the slower growth and evolution of irradiationinduced defects compared to the single component material was attributed to a suppressed diffusion effect. The irradiation of a quaternary $\mathrm{Fe}_{27} \mathrm{Cr}_{18} \mathrm{Mn}_{27} \mathrm{Ni}_{28} \mathrm{CSA}$ (at.\%) with 3 and $5.8 \mathrm{MeV} \mathrm{Ni}$ ions in the temperature range from 673 to $973 \mathrm{~K}$ showed that the FCC phase did not exhibit any phase transformation up to doses around $10 \mathrm{dpa}$, but it was observed that radiation-induced segregation (RIS) at grain boundaries is significantly suppressed when compared to FeCrNi and FeMnCr conventional alloys $[29,30]$. All these recent works are suggesting a remarkably high radiation resistance of the studied CSAs, although the underlying physical mechanisms for such enhancement are still a subject of investigation.

As a result of the CSAs chemical complexity, some authors have recently proposed that reduced defect mobility (commonly referred to as sluggish diffusion) and modified system energetics are key mechanisms responsible for the superior radiation resistance in these alloys [20,31,32]. Ion irradiation with 1.5 and $3 \mathrm{MeV} \mathrm{Ni}^{+}$ were performed for pure $\mathrm{Ni}, \mathrm{NiCo}, \mathrm{NiFe}$ and $\mathrm{NiCoFeCr}$ up to a fluence of $5 \times 10^{16}$ ions $\cdot \mathrm{cm}^{-2}$ (corresponding to a peak damage dose of $60 \mathrm{dpa}$ ). The authors' conclusions on reduced mobility of defects were based on the experimental evidence that the areal density and size distribution of radiation-induced voids were considerably smaller for the $\mathrm{NiCoFeCr}$ CSA when compared with pure $\mathrm{Ni}$ [22]. These results were further elucidated by recent computational research involving molecular dynamics (MD) and ab initio methods based on density functional theory (DFT), as recently reviewed by Zhao et al. [33,34]. When compared with pure metals and dilute alloys, CSAs would exhibit (i) wide defect energy distributions instead of specific values, (ii) different migration barriers that depend on the local configuration, (iii) efficient recombination dynamics of Frenkel pairs (enhanced recombination volume) that result in a "self-healing" effect under irradiation and (iv) elemental-biased diffusion and mass transport.

A recent investigation using the Cluster Variation Method (a statistical mechanics method used for modelling multicomponent simple solid solutions) investigated the constituting hypotheses in the field of HEAs at a fundamental level $[4,7]$. It was shown that the configurational entropy has only a small (if any) effect on solid solution stability and in particular it was demonstrated that, in selected cases, the configurational entropy of the solid solution at the onset of long-range ordering may proportionally decrease as the number of components in an equiatomic solid solution increase [4]. The stabilising effect of CSAs was detected, but it was attributed to the existence of conflicting interactions between the alloy components or "con- 
figurational frustration". Furthermore, this work also investigated whether there is some possible thermodynamic base for an ad hoc sluggish diffusion effect in these concentrated solid solutions. The results suggested that these alloys behave as any concentrated solid solution. Moreover, the thermodynamic effect of cross-diffusion coefficients (the coefficients that describe the diffusion flux of one component, affected by the gradient of chemical potential of another) may be as strong, or even stronger than the effect on the direct diffusion coefficients (i.e. the coefficients which control the diffusion flux of a components, affected by the gradient of its own chemical potential). This points to a high complexity of diffusion in these concentrated alloys, and it is probable that one of the manifestations of this complexity is the "sluggish diffusion" effect.

In this work, in order to probe the reduced mobility concept of irradiationinduced defects in CSAs, a FeCrMnNi CSA and an austenitic stainless steel (the AISI-348 steel) were subjected to light and heavy ion irradiation under equivalent irradiation and annealing conditions. Their microstructural responses were monitored in situ within a transmission electron microscope (TEM). Further experimental evidence that this CSA has suppressed/delayed defect energetics and mobility under irradiation, using existing theories of bubble and void nucleation and growth $[35,36]$ in solids is presented.

\section{Experimental Methods}

\subsection{Sample preparation}

The bulk CSA FeCrMnNi was cast at the Oak Ridge National Laboratory using an arc-melting furnace and drop casting method. Detailed synthesis routes for this alloy can be found elsewhere [29]. The austenitic stainless steel AISI-348 is a commercial alloy. TEM samples were produced from the bulk alloys by using a Focused Ion Beam (FIB) system and mounted onto Mo grids using the conventional lift-out technique [37]. Energy dispersive X-ray spectroscopy (EDX) was used to determine the composition of the alloys using an FEI Versa 3D Dual-Beam. The composition of the as-received alloys were determined by means of the standardless Cliff-Lorimer quantification method [38] and are shown in Table 1. The AISI-348 steel alloy also contained minor additions of elements like $\mathrm{Nb}, \mathrm{Si}$ and $\mathrm{C}$ at the levels of 0.32, 0.36 and $0.037 \mathrm{wt} \%$, respectively.

\subsection{Light and heavy ion irradiation in situ within a TEM}

TEM analyses with in situ ion irradiation experiments were performed at the MIAMI1 and -2 facilities at the University of Huddersfield with $134 \mathrm{keV} \mathrm{Xe}^{+}$and $6 \mathrm{keV} \mathrm{He}^{+}$ 
Table 1: Primary elemental composition of the austenitic stainless steel AISI-348 and the FeCrMnNi CSA measured by SEM-EDX (error is $\approx 3 \%$ ).

\begin{tabular}{|c|c|c|}
\hline Element & AISI-348 steel [wt.\%] & HEA [wt.\%] \\
\hline $\mathbf{F e}$ & Balance $^{\dagger}$ & 26.80 \\
\hline $\mathbf{C r}$ & 18.08 & 18.40 \\
\hline $\mathbf{N i}$ & 9.38 & 27.50 \\
\hline $\mathbf{M n}$ & 1.10 & 27.30 \\
\hline
\end{tabular}

†The AISI-348 steel has minor alloying elements (C, Si, S, P, Nb, Co and Ta) in which the sum of the content is $<1$ wt.\%.

ions. The ion energies were chosen so that the majority of these inert gas species will be retained in the TEM specimens based on SRIM calculations. Implantations were performed at room temperature in order to minimise bulk diffusion. MIAMI-1 consists of a JEOL JEM-2000FX TEM coupled with a $100 \mathrm{kV}$ ion accelerator, while MIAMI-2 couples a Hitachi H-9500 TEM with a $300 \mathrm{kV}$ ion accelerator [39]. The energies of the light and heavy ions were selected to approximate both implanted ion range and damage profile as predicted from SRIM quick Kinchin-Pease damage calculations (see section 2.3). Details on the design of the experiment are presented in figure $1(a-b)$.

After the irradiation, the samples were annealed up to $673 \mathrm{~K}$ for approximately 40 minutes using a Gatan double-tilt heating holder. The evolution of their microstructures was monitored in situ within the TEM by means of a Gatan ORIUS SC200 digital camera. A heating ramp of $60 \mathrm{~K}$ per minute was used to reach the temperature of $673 \mathrm{~K}$.

Gas bubbles were observed in bright field (BFTEM) mode with the objective lens underfocused by approximately $1000 \mathrm{~nm}$ in order to obtain well-delineated images of the bubbles (Fresnel contrast) [36]. Bubble sizes were measured using the software ImageJ [40] with protocols already reported in literature [41, 42].

\subsection{Fluence-to-dpa conversion and SRIM calculations}

The Monte-Carlo code Stopping and Range of Ions in Matter (SRIM-2013) [43] was used with the Kinchin-Pease model in order to carry out fluence-to-dpa conversion as suggested by Stoller et al. [44]. In our calculations, the displacement energy for each alloying element was set to $40 \mathrm{eV}$ based on reference values [45] and the samples thicknesses were assumed to be within the range of 70 to $75 \mathrm{~nm}$. Samples were irradiated using $134 \mathrm{keV}$ Xe up to a fluence of $2.7 \times 10^{15}$ ions $\cdot \mathrm{cm}^{-2}$ (corresponding to approximately $4 \mathrm{dpa}$ ), and $6 \mathrm{keV} \mathrm{He}$, up to a maximum fluence of $1.7 \times 10^{17}$ ions $\cdot \mathrm{cm}^{-2}$ (or $4 \mathrm{dpa}$ ). The fluence-to-dpa profile is shown in the figure 1(c). 

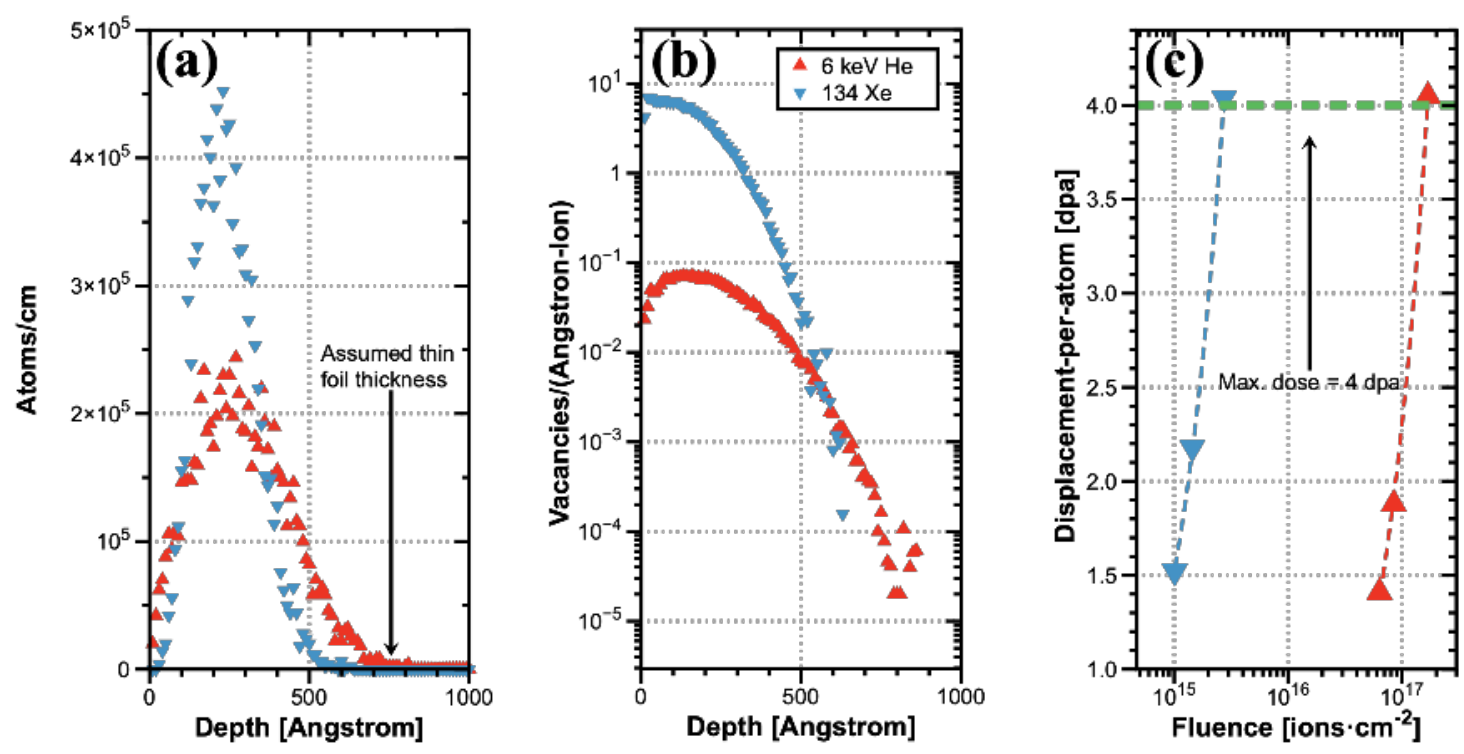

Figure 1: SRIM-2013 calculations of (a) implanted ranges, (b) damage profiles and (c) fluence-todpa conversion for both alloys irradiated with $6 \mathrm{keV}$ He and $134 \mathrm{keV}$ Xe ions. The Monte Carlo calculations with SRIM-2013 led to an equivalent number of displacements per ion collision in both AISI-348 steel and FeCrMnNi CSA.

\section{Results}

The experiments were designed to investigate the microstructural response of both the FeCrMnNi CSA and the AISI-348 steel in situ in the TEM under the same irradiation conditions. The results are subdivided with respect to the ion species and energies.

\subsection{Light ion irradiation at room temperature and subsequent annealing}

The microstructure of the FeCrMnNi before irradiation with $6 \mathrm{keV}$ He ions is shown in the BFTEM micrograph 2(a). He bubbles were first observed (resolvable) in the CSA at a fluence of $7.1 \times 10^{16} \mathrm{ions} \cdot \mathrm{cm}^{-2}$ (or $1.5 \mathrm{dpa}$ ) and became clearly observable throughout the matrix and trapped at interfaces up to 4 dpa (figure $2(\mathrm{~b}-\mathrm{c})$ ). The damage microstructure of the CSA after subsequent annealing is presented in figure 2(d) where He bubbles exhibited preferential growth at interfaces. The diffraction pattern inset of figure 2(d) shows (a FCC crystal structure) that the FeCrMnNi CSA has not suffered phase decomposition, suggesting that the material remains as a disordered solid solution single phase matrix.

Similarly, the microstructure of the AISI-348 steel before irradiation is shown in figure 3(a), in which annealing twins are observed in the austenite matrix. He bubbles are observed in the austenitic stainless steel under irradiation up to a fluence of $3.7 \times 10^{16}$ ions $\cdot \mathrm{cm}^{-2}$ (or $0.83 \mathrm{dpa}$ ). The damaged microstructure after $6 \mathrm{keV} \mathrm{He}$ ion irradiation up to $4 \mathrm{dpa}$ is shown in figure $3(\mathrm{~b})$ where He bubbles were observed 


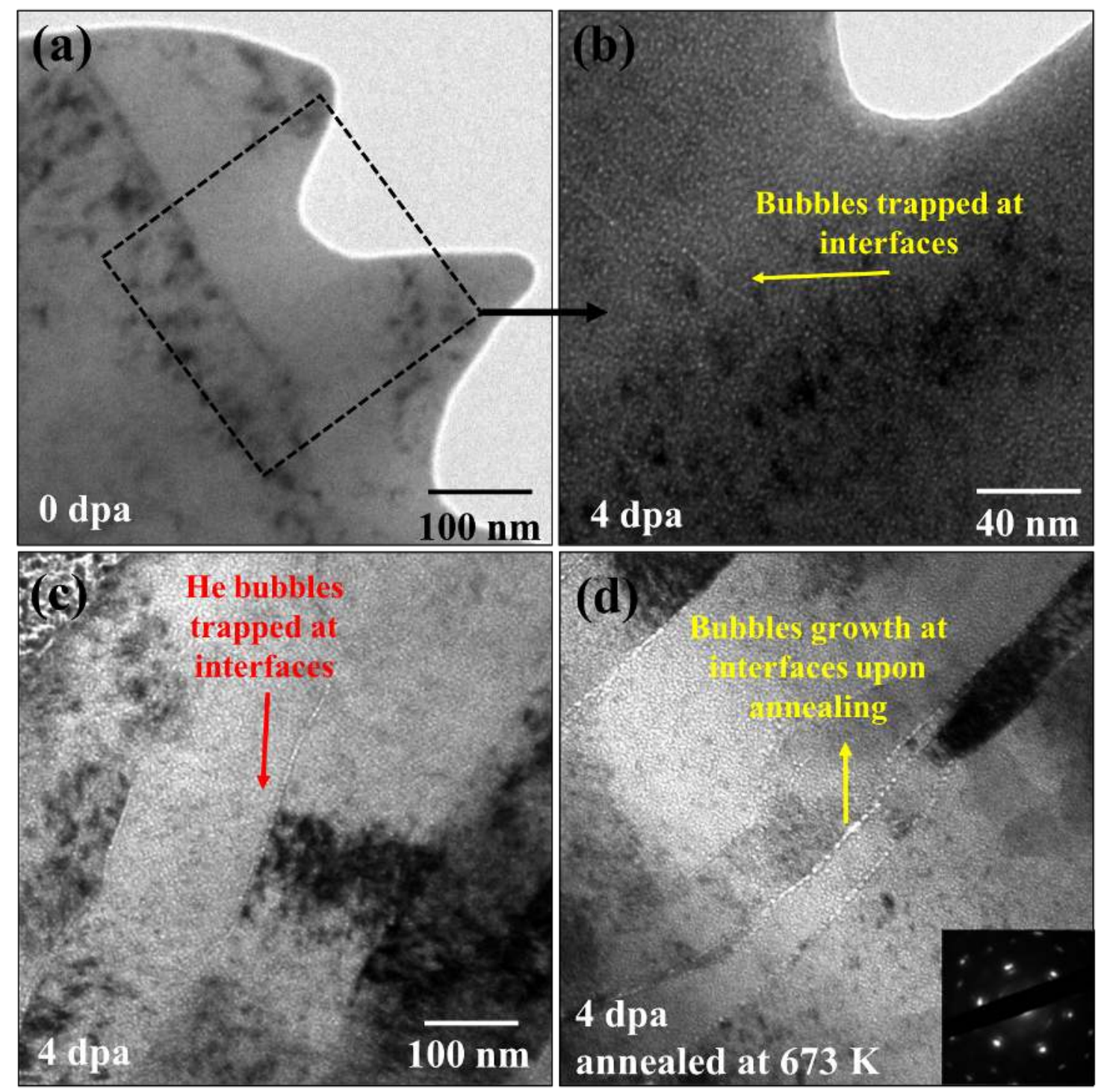

Figure 2: Underfocused BFTEM micrographs of the FeCrMnNi CSA (a) before irradiation and (b-c) after $6 \mathrm{keV}$ He irradiation up to $4 \mathrm{dpa}$. Microstructure of the alloy after $4 \mathrm{dpa}$ and subsequent annealing at $673 \mathrm{~K}(\mathrm{~d})$, where the inset represents the diffraction pattern of that area after irradiation and annealing. Note: the scale marker in (c) also applies to (d). 
in the austenite matrix at $4 \mathrm{dpa}$, but not at the interfaces between the matrix and annealing twins. After $4 \mathrm{dpa}$ and subsequent annealing at $673 \mathrm{~K}$, bubbles were observed to grow in the matrix as exhibited in figure $3(\mathrm{c}-\mathrm{d})$.

He bubbles were observed to be trapped at the grain boundaries in the AISI-348 steel after $4 \mathrm{dpa}$ as showed in figure 4(a) both after irradiation and after annealing, the bubbles were also observed to grow at these interfaces as shown in figures 4(b-d). As confirmed by the diffraction pattern inset of figure $3(\mathrm{~d})$, the austenite phase has not suffered decomposition. For all the irradiation and annealing experiments, the electron beam appears to have a minimal apparent influence on the nucleation and growth of inert gas bubbles and this was confirmed by analysing areas that were not exposed to the electron beam during the ion irradiation experiments.

\subsection{Heavy ion irradiation at room temperature and subsequent annealing}

The microstructure of the FeCrMnNi CSA before the $134 \mathrm{keV}$ Xe ion irradiation is shown in figure 5(a). During irradiation, Xe bubbles became observable in the matrix of the FeCrMnNi CSA at a fluence around of $4.8 \times 10^{14}$ ions $\cdot \mathrm{cm}^{-2}$ (or 0.73 dpa) and after $4 \mathrm{dpa}$, Xe bubbles were observed both in the matrix and trapped at interfaces, as shown in figure 5(b-c). Subsequent annealing at $673 \mathrm{~K}$, figure $5(\mathrm{~d})$, revealed that the Xe bubbles have not undergone significant alteration when compared to those just prior to the annealing. The inset in figure $5(\mathrm{~d})$ shows that the CSA has not suffered phase decomposition within these experimental conditions.

Correspondingly, the microstructure of the AISI-348 steel before the $134 \mathrm{keV}$ Xe ion irradiation is shown in figure 6(a) in which annealing twins were present in the austenite matrix. Xe bubbles became observable in the austenite matrix under irradiation at a fluence of $1.0 \times 10^{15} \mathrm{ions} \cdot \mathrm{cm}^{-2}$ (or $1.5 \mathrm{dpa}$ ). The annealing twins were dissolved after irradiation up to $4 \mathrm{dpa}$ (confirmed by tilting the specimen) and Xe bubbles were trapped both at grain boundaries as well as in the austenite matrix, as shown in figure $6(\mathrm{~b}-\mathrm{c})$. After subsequent annealing at $673 \mathrm{~K}$ - figures $6(\mathrm{e}-\mathrm{f})$ - Xe bubbles have grown preferentially in the matrix as shown in figure $6(\mathrm{~g})$.

\subsection{Bubble size determination}

By measuring the diameter of $\mathrm{He}$ and $\mathrm{Xe}$ bubbles that have nucleated and grown after irradiation up to $4 \mathrm{dpa}$ and subsequent annealing at $673 \mathrm{~K}$, size distribution statistical analysis was performed for the alloys under investigation. The study of nucleation and evolution of in inert gas bubbles in the CSA and AISI-348 steel was subdivided for bubbles in the matrix and trapped at the interfaces with the results summarised in figure 7 , exhibiting the size distribution histograms for each case as well as the average diameter size values and the associated standard deviation. 


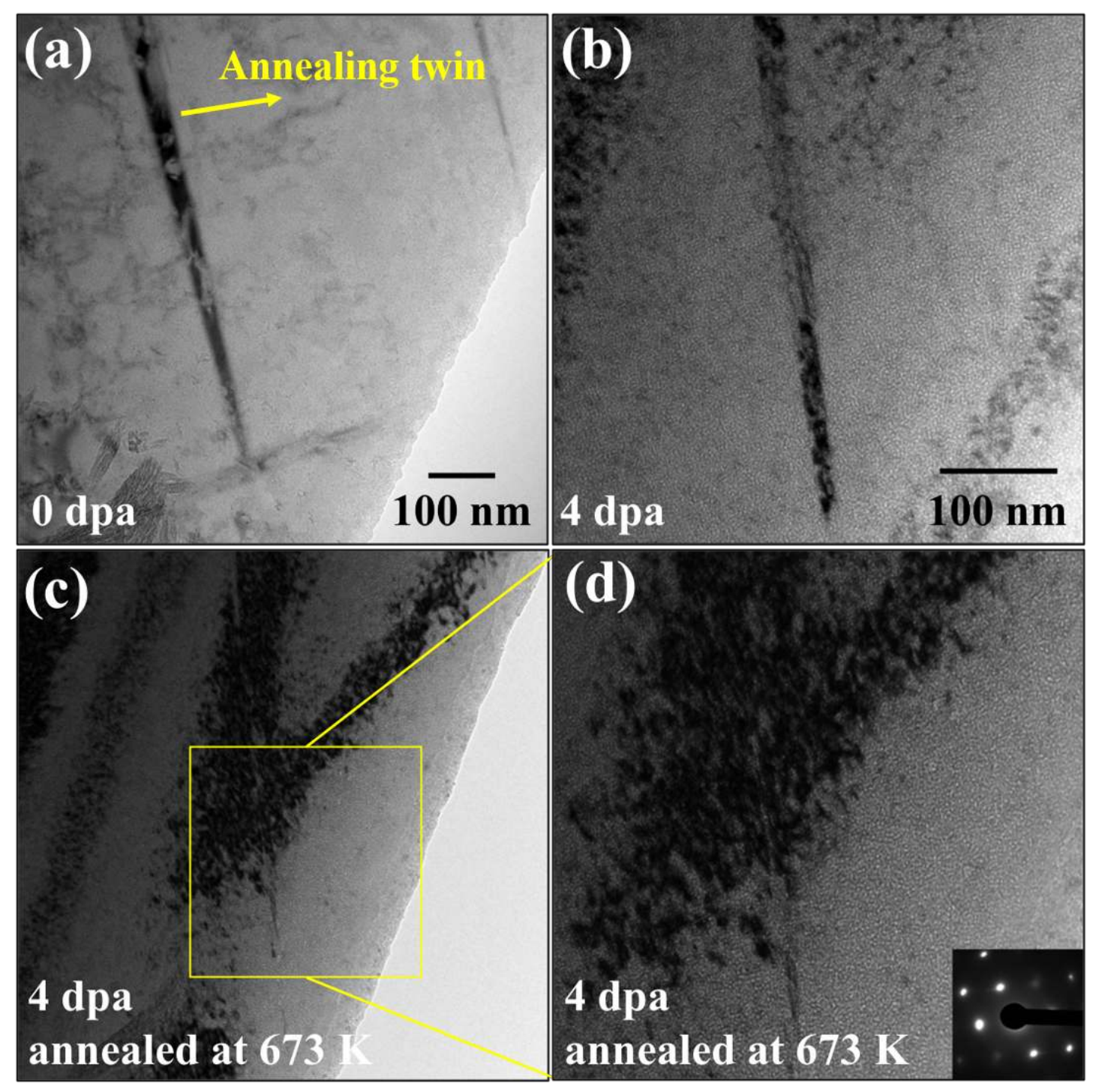

Figure 3: Underfocused BFTEM micrographs showing the microstructure of the AISI-348 steel (a) before irradiation and (b) after $6 \mathrm{keV}$ He irradiation up to 4 dpa. The micrographs (c) and (d) show the alloy after $4 \mathrm{dpa}$ and subsequent annealing at $673 \mathrm{~K}$. The inset to (d) shows the diffraction pattern of that area after irradiation and annealing. Note: the scale marker in (a) also applies to (c) and the scale marker in (b) also applies to (d). 


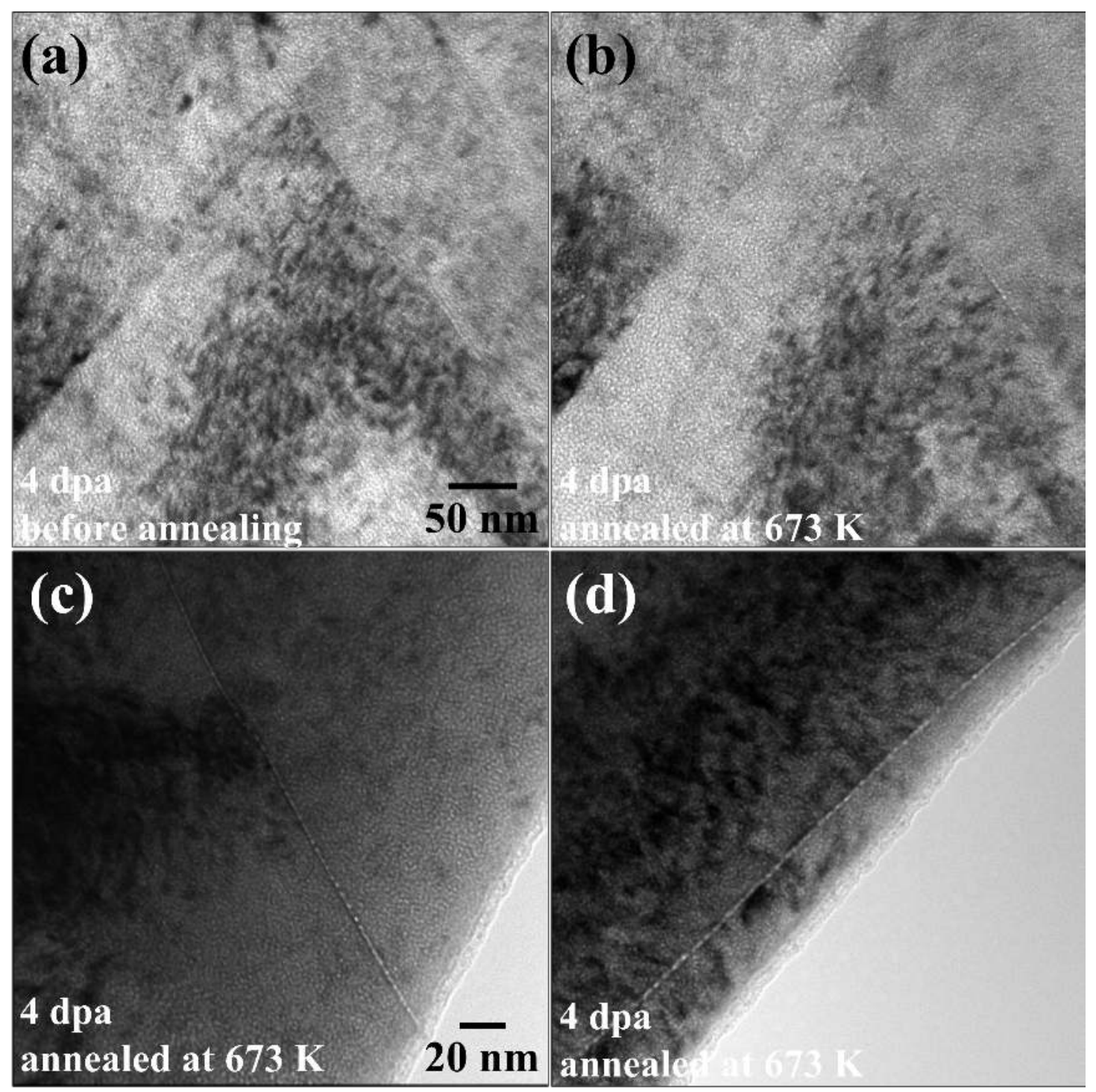

Figure 4: BFTEM micrographs of the AISI-348 steel irradiated with 6 keV He up to 4 dpa showing (a) He bubbles trapped at interfaces before annealing and (b-d) after annealing at $673 \mathrm{~K}$. The micrographs (c) and (d) show that such accumulation effect was also detected at interfaces that were not under constant electron beam exposure. Note: scale marker in (a) also applies to (b) and the scale marker in (c) also applies to (d). 

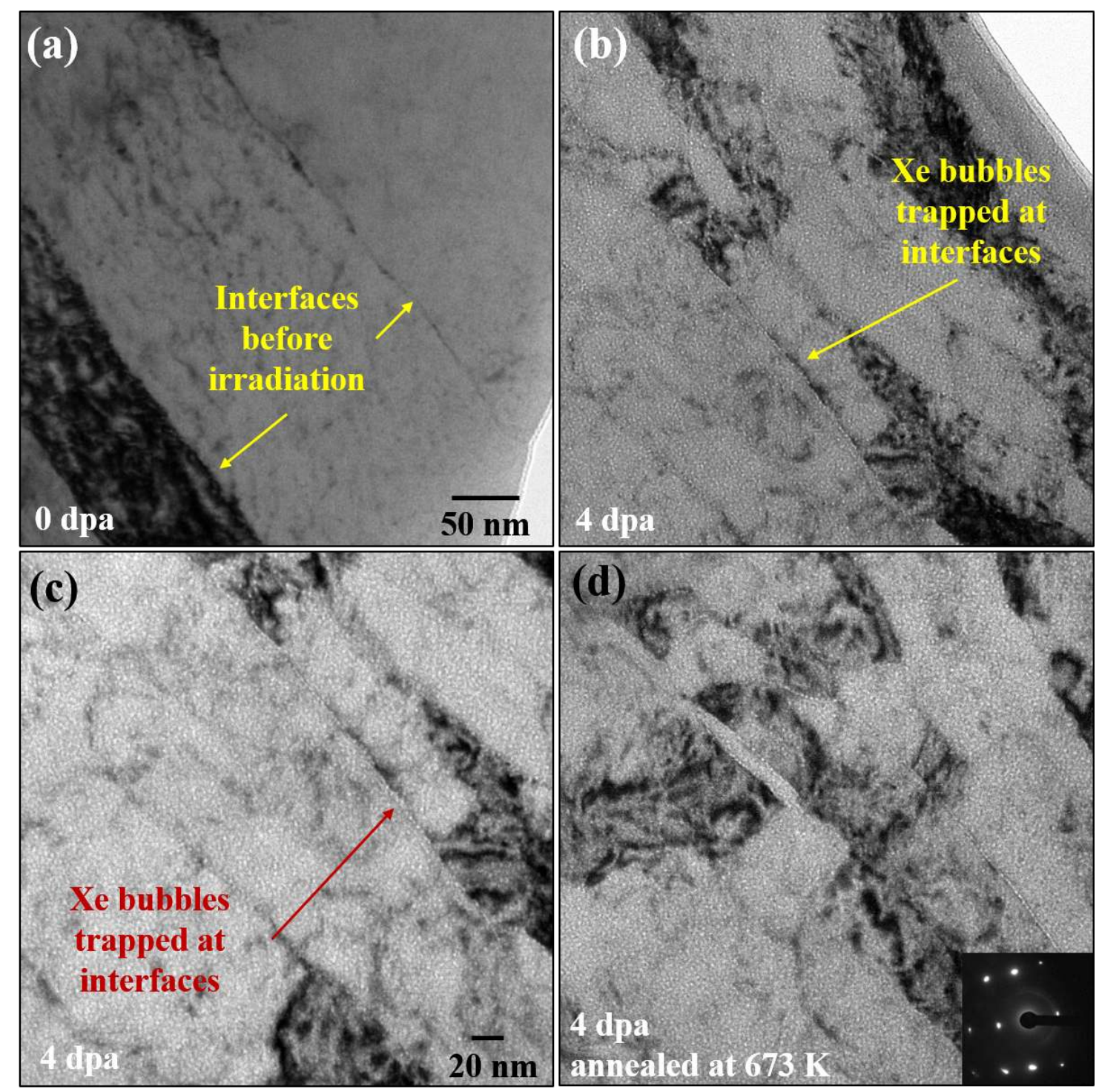

Figure 5: BFTEM micrographs of the FeCrMnNi CSA (a) before irradiation and (b-c) after 134 $\mathrm{keV}$ Xe irradiation up to 4 dpa. The micrograph (d) exhibit the damaged microstructure after subsequent annealing at $673 \mathrm{~K}$. The inset in (d) is the diffraction pattern of that area after irradiation and annealing. Note: the scale marker in (a) also applies to (b) and the scale marker in (c) also applies to (d). 


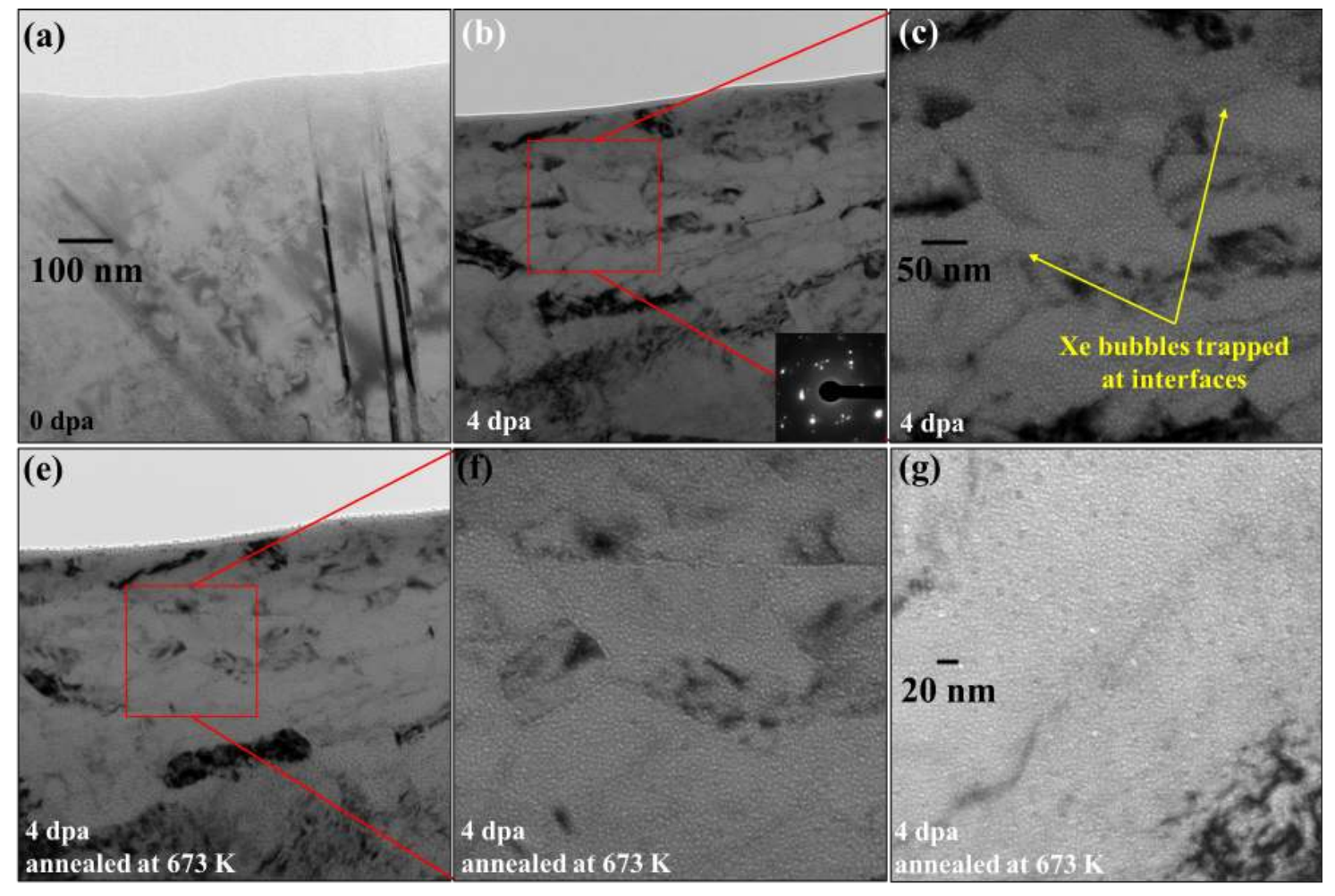

Figure 6: BFTEM micrographs of the AISI-348 steel (a) before irradiation and (b-c) after 134 $\mathrm{keV} \mathrm{Xe}$ irradiation up to $4 \mathrm{dpa}$. The microstructure after annealing at $673 \mathrm{~K}$ is shown in the micrographs (e-g). Note: the scale marker in (a) also applies to (b) and (e) and the scale marker in (c) also applies to (f). 
$6 \mathrm{keV} \mathrm{He}$
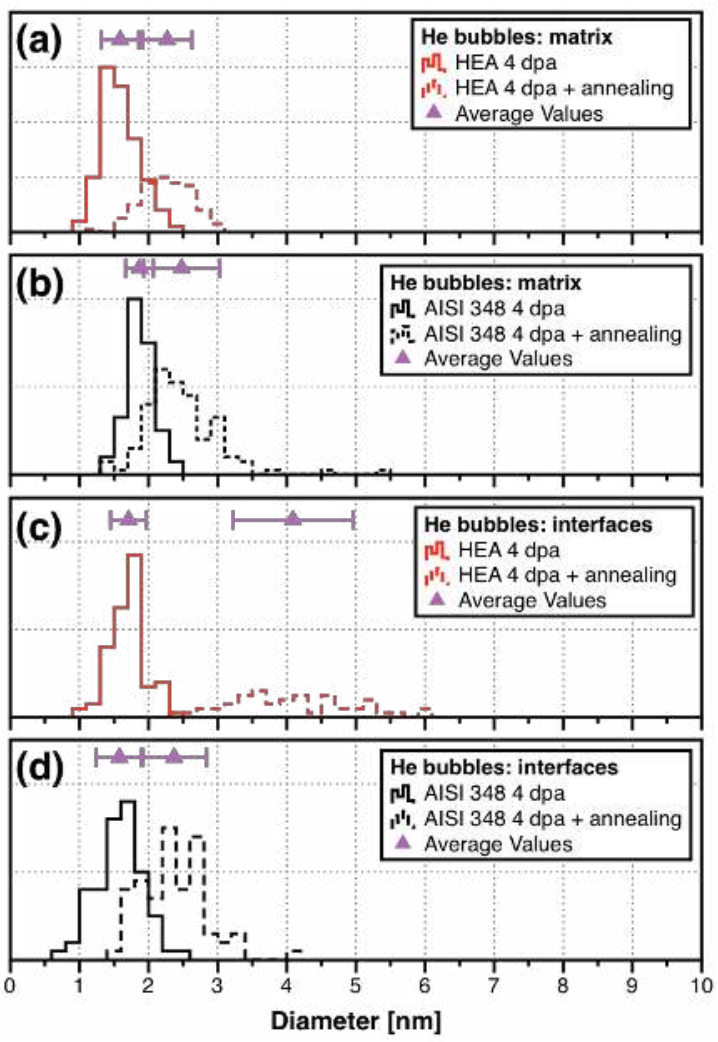

$134 \mathrm{keV}$ Xe
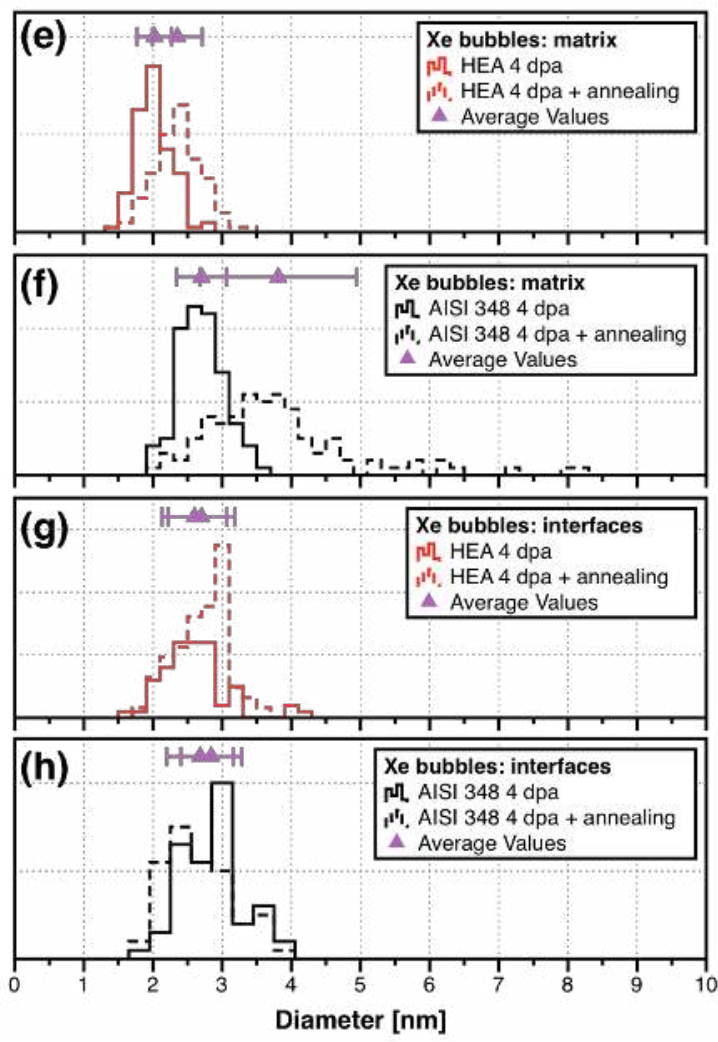

Figure 7: Size (diameter) distributions of He and Xe bubbles in the matrix and at the interfaces of both the FeCrMnNi CSA and the AISI-348 steel after irradiation up to 4 dpa and subsequent annealing at $673 \mathrm{~K}$. Note: the error bars are the standard deviations of each data set. 


\section{Discussion}

The physical mechanisms for inert gas bubbles nucleation and growth in crystalline solids has been a subject of intense study over the past decades and are intrinsically related to the kinetics of point defects at the atomic level. It is well known that inert gas atoms have low solubility in metals $[46,47]$. In a crystalline solid, with mobile vacancies and implanted inert gas atoms, bubbles will grow by the incorporation of vacancies and inert gas atoms interstitials to the cavity. Similarly, they will shrink upon vacancy emission. A steady-state equilibrium condition will be established when the rates of absorption and departure of vacancies are equal [36, 48]. By using such concepts, the current hypothesis on the reduced mobility of defects in CSAs can be tested by analysing the behaviour of inert gas bubbles in the microstructure of the FeCrMnNi CSA.

As a result of the ion irradiation in FeCrMnNi CSA and AISI-348 steel, Frenkel pairs will be generated in a cascade of defects. Monte Carlo calculations using SRIM-2013 indicates that $6 \mathrm{keV}$ He ion irradiation generates approximately 10 vacancies per ion collision on average for both alloys whilst $134 \mathrm{keV}$ will generate approximately 900 vacancies per ion collision - an increase in damage injection of nearly two orders of magnitude higher by much heavier Xe ions. Upon the cooling down of the ballistic phase (in a time frame of a half picosecond) of the damage cascade [49], defect recombination will take place during the kinetic phase within a picosecond [49] and only a fraction $(\approx 30 \%$ for metals $[50,51])$ of the initial defect distribution will survive. Multi-scale modelling indicates that these atomic-driven processes of defect generation and recombination due to irradiation extends to the nanosecond timescale [52], sluggish diffusion and defect evolution can continue even into microseconds [53]. The surviving defects will eventually recombine with the implanted inert gases interstitial atoms and bubbles will nucleate throughout the microstructure.

The irradiation performed with He shows that He bubbles have nucleated in the matrix of both alloys at room temperature, and at doses of up to 4 dpa the average diameter for He bubbles is slightly larger in the AISI-348 steel compared with the FeCrMnNi CSA. After annealing, the He bubbles have grown approximately $42 \%$ (AISI-348 steel) and 33\% (FeCrMnNi CSA) in diameter. Given that He atoms in metals are mobile at $673 \mathrm{~K}[47]$ and with respect to He bubbles in the matrices of both alloys after irradiation and extended annealing, the results suggest on a trending basis that the growth mechanism of inert gas bubbles in the CSA may be slightly supressed. The effect of $6 \mathrm{keV}$ He irradiation in these alloys led to very similar results in both alloys when the statistical error is considered. It is 
worth emphasising that, as per the SRIM-2013 calculations, the number of vacancies generated in a single $6 \mathrm{keV}$ He ion collision is small and after recombination, the reduced number of remaining defects will be a limiting factor for the growth of He bubbles in the matrices of these alloys.

On the other hand, He bubbles were observed to grow significantly at the interfaces of the FeCrMnNi CSA as exhibited in figure 1(d) and the distribution in figure $7(\mathrm{c})$. The same growth effect has not been observed to the same extent for the AISI-348 steel. These experimental observations suggest that the interfaces as grain and twin boundaries present in the CSA microstructure are prone to act as sinks for irradiation-induced defects upon annealing. At first glance the effects of 6 $\mathrm{keV}$ He ion irradiation in the FeCrMnNi CSA exhibits a discrepancy: upon annealing, bubbles in the FeCrMnNi CSA do not grow significantly in the matrix, but are prone to grow at the interfaces.

Such experimental observations can be explained with the most recent works in CSAs. For these complex alloys, computer simulations within the scope of molecular dynamics and density functional theory have suggested that both the formation energies for point defects and the migration barriers for diffusion are higher in CSAs compared with pure metals and dilute alloys [33]. As a result, on the nucleation and evolution of He bubbles in the matrix of the FeCrMnNi CSA, it is reasonable to assume that the observed trend of reduced growth (when compared with the AISI348 steel) can be apparently explained by the sluggish diffusion effect which is also intrinsically related with an enhanced recombination effect (self-healing), leading to a reduced generation and survival distribution of primary collisional defects as recently proposed by several authors [22, 31-33, 49, 54].

The complex energy landscape and the subsequent sluggish defect kinetics property characteristic of concentrated solid solution alloys are strongly affected by temperature according to Zhao et al. [31, 33]: at temperature close to the homologous temperature, the sluggish diffusion property is assumed to cease. Osetsky et al. [53] have also pointed out that the physical origin of the sluggish diffusion effect may be attributed to the compositional dependence of vacancy migration energy with maximum near the site percolation threshold, and argued that the coupled percolation and composition-dependent barriers for vacancy jumps within different subsystems in CSAs leads to the sluggish diffusion [53].

Another factor affecting the reduced mobility of defects in CSAs is the presence of interfaces by inducing preferential diffusion of some chemical species and point defects at these site specific dependencies. Preferential channels for diffusion of interstitials and vacancies will exist and they will be dominant near interfaces. As 
a result of this effect, chemical segregation processes have been reported to occur in some CSAs under irradiation [29], although this has not been observed for He irradiations on the FeCrMnNi CSA in this present work: if significant radiationinduced segregation had occurred, electron-contrast differences would be observed along the interfaces of the studied alloys as previously reported by our group in similar irradiation conditions [55]. These facts can explain the anomalous growth of He bubbles at the interfaces of the FeCrMnNi CSA upon subsequent annealing as herein reported and in contrast with the observed results in the matrix. In the stainless steel, the same effect has not been observed to the same extent, possibly because in this material, the tendency is for the He bubbles to grow in the matrix thus reducing the population of surviving defects that could be available to diffuse towards the interfaces. Therefore, these mechanisms require further investigation.

With respect to the irradiation with $134 \mathrm{keV} \mathrm{Xe,} \mathrm{the} \mathrm{same} \mathrm{trends} \mathrm{observed} \mathrm{in}$ the He irradiation are reproduced. The bar plot in figure $7(\mathrm{c})$ shows that after the heavy ion irradiation up to $4 \mathrm{dpa}$, Xe bubbles are larger in the austenite matrix in the stainless steel when compared with the CSA. Upon annealing, the growth is also more prominent in the steel. A single $134 \mathrm{keV}$ Xe ion collision will generate approximately $10^{3}$ vacancies. Following the postulated reduced mobility of defects and the enhanced recombination effect, the delayed growth of Xe bubbles in the CSA is consistent with the results observed with $6 \mathrm{keV} \mathrm{He}$, but if the statistical error is again taken into consideration, the radiation response of both alloys under exposure to energetic heavy ions will lead to similar results. At the interfaces, figure $7(\mathrm{~d})$, Xe bubbles have not grown significantly in both materials and this can be correlated with the reduced mobility of Xe atoms $[56,57]$ under the experimental conditions and temperatures in the performed experiments.

Assuming the similarities of the results in both alloys and, in particular, the statistical error associated with the bubbles size distribution, defect population and availability as well as inert gas atomic mobilities are factors under consideration to interpret the observed trending. Figure 7 establishes a baseline: (i) light ion irradiations lead to similar bubbles sizes in the matrix of both alloys, but significant growth at the interfaces in the FeCrMnNi CSA and (ii) heavy ion irradiations lead to equivalent bubbles at the interfaces, but significant growth in the matrix of the AISI-348 steel.

Heavy ion irradiation will generate two orders of magnitude more defects than light ion irradiation which imply in vastly different cascade structures. By this, He irradiation will likely result in Frenkel pairs whereas the Xe irradiation will result in small defect clusters that are somewhat (meta-)stable. As a result, tie-up of defects 
can readily occur in the Xe case. On the inert gases mobilities, He can migrate through the lattice of the alloys more easily than Xe (which is not very mobile at the studied temperatures). If the He bubbles in the CSA interfaces grow by incorporation of He, with minimal impact on the diffusion of defects (vacancies), Xe may be more mobile in the AISI-348 steel and will be able to interact with the small defect clusters in the matrix resulting in larger bubbles as observed. In this way, the hypothesis of sluggish diffusion can be used to explain the observed trending in the bubble sizes.

\section{Conclusions}

Herein by analysing trends in data regarding the nucleation and growth of inert gas bubbles in a concentrated solid solution alloy, the FeCrMnNi CSA, and an intermediary solid solution alloy, the austenitic stainless steel AISI-348, at the equivalent irradiation and annealing conditions, the following conclusions:

- Light ion irradiation has led to similar bubbles sizes in the bulk matrix, but growth at the interfaces of the FeCrMnNi CSA. Heavy ion irradiation has led to equivalent bubbles in the interfaces, but growth in the matrix of the AISI-348 steel.

- Due to differences in defect populations and collision cascades morphology, He irradiations are likely to generate Frenkel pairs whereas Xe irradiations can result in small defect clusters that will induce tie-up of defects in the Xe case compared with the He case. At the studied temperatures, the fast mobility of $\mathrm{He}$ and reduced mobility of Xe, He bubbles grow at the interfaces of the FeCrMnNi CSA via He incorporation with minimal impact on the diffusion of vacancies. Similarly, Xe may be more mobile in the AISI-348 steel and can interact with the small clusters of defects resulting in larger bubbles in the bulk matrix when compared with the results in the FeCrMnNi CSA.

- Trending on data indicates that He bubbles have grown faster in the matrix of the AISI-348 steel compared with in the FeCrMnNi CSA. After annealing, He bubbles in the matrix have also grown larger in the first alloy. Similar results were obtained by irradiating both alloys with the heavier ions. These results show that the complex thermodynamic state of the FeCrMnNi CSA may act in order to limit or delay the growth of inert gas bubbles by suppressing the diffusion of point defects in the matrix of the nearly equiatomic alloy. It is worth emphasising that in this sense, the "sluggish" diffusion is a result of 
the complex energy landscape of CSAs in agreement with the most recent understanding of the scientific community.

When the statistical error on measuring the sizes of the inert gas bubbles is taken into account, the radiation response at lower doses for both alloys can be considered to be very similar and in this sense the sluggish diffusion effect plays only a minor role. The observation of inert gas bubbles accumulation at the interfaces may diminish the applicability of this CSAs within the context of future nuclear reactors as they play key roles in embrittlement and swelling resulting in strong degradation of mechanical properties in many materials and alloys, but the occurrence of inert gas bubbles and their relation with mechanical properties in CSAs are still subjects of future research.

\section{Acknowledgements}

MAT was supported through ASTRO, a United States Department of Energy workforce development program implemented at Oak Ridge National Laboratory through the Oak Ridge Institute for Science and Education under contract number DEAC05-06OR23100. YZ and HB were supported as part of the Energy Dissipation to Defect Evolution (EDDE), an Energy Frontier Research Center funded by the U.S. Department of Energy, Office of Science, Basic Energy Sciences under contract number DE-AC05-00OR22725. CGS acknowledges the financial support by the São Paulo State Research Funding Agency (FAPESP, São Paulo, Brazil) under grant 2016/25248-3. All the authors are grateful to the Engineering and Physical Sciences Research Council (EPRSC) for funding MIAMI-1 and MIAMI-2 facilities (grant numbers EP/E017266/1 and EP/M028283/1).

\section{References}

[1] B. Cantor, K. Kim, P. J. Warren, Novel Multicomponent Amorphous Alloys, Materials Science Forum 386-388 (2002) 27-32.

[2] B. Cantor, Multicomponent and High Entropy Alloys, Entropy 16 (9) (2014) 4749-4768.

[3] B. Murty, J. Yeh, S. Ranganathan, High-Entropy Alloys, 2014.

[4] C. G. Schön, T. Duong, Y. Wang, R. Arróyave, Probing the entropy hypothesis in highly concentrated alloys, Acta Materialia 148 (2018) 263-279.

[5] D. B. Miracle, O. N. Senkov, A critical review of high entropy alloys and related concepts, Acta Materialia 122 (2017) 448-511.

[6] B. Kombaiah, K. Jin, H. Bei, P. D. Edmondson, Y. Zhang, Phase stability of single phase Al 0 . 12 CrNiFeCo high entropy alloy upon irradiation, Materials and Design 160 (2018) $1208-1216$. 
[7] C. G. Schon, T. Duong, Y. Wang, R. Arroyave, A proof of concept: Thermodynamics of aluminum-transition metal highly concentrated alloys, Journal of Alloys and Compounds 781 (2019) 595-605.

[8] Y. Zhang, T. T. Zuo, Z. Tang, M. C. Gao, K. A. Dahmen, P. K. Liaw, Z. P. Lu, Microstructures and properties of high-entropy alloys, Progress in Materials Science 61 (2014) 1-93.

[9] Y. Ye, Q. Wang, J. Lu, C. Liu, Y. Yang, High-entropy alloy: challenges and prospects, Materials Today 19 (6) (2016) 349-362.

[10] Z. J. Zhang, M. M. Mao, J. Wang, B. Gludovatz, Z. Zhang, S. X. Mao, E. P. George, Q. Yu, R. O. Ritchie, Nanoscale origins of the damage tolerance of the high-entropy alloy CrMnFeCoNi, Nature Communications 6 (1) (2015) 10143.

[11] M. Vaidya, K. Pradeep, B. Murty, G. Wilde, S. Divinski, Bulk tracer diffusion in cocrfeni and cocrfemnni high entropy alloys, Acta Materialia 146 (2018) 211-224.

[12] C. Ng, S. Guo, J. Luan, S. Shi, C. T. Liu, Entropy-driven phase stability and slow diffusion kinetics in an al0. 5cocrcufeni high entropy alloy, Intermetallics 31 (2012) 165-172.

[13] E. Pickering, N. G. Jones, High-entropy alloys: a critical assessment of their founding principles and future prospects, International Materials Reviews 61 (3) (2016) 183-202.

[14] W. Kucza, J. Dkabrowa, G. Cieslak, K. Berent, T. Kulik, M. Danielewski, Studies of sluggish diffusion effect in co-cr-fe-mn-ni, co-cr-fe-ni and co-fe-mn-ni high entropy alloys. determination of tracer diffusivities by combinatorial approach, Journal of Alloys and Compounds 731 (2018) 920-928.

[15] D. Gaertner, J. Kottke, G. Wilde, S. V. Divinski, Y. Chumlyakov, Tracer diffusion in single crystalline cocrfeni and cocrfemnni high entropy alloys, Journal of Materials Research (2018) $1-8$.

[16] W. Chen, L. Zhang, High-throughput determination of interdiffusion coefficients for co-cr-femn-ni high-entropy alloys, Journal of Phase Equilibria and Diffusion 38 (4) (2017) 457-465.

[17] R. Wang, W. Chen, J. Zhong, L. Zhang, Experimental and numerical studies on the sluggish diffusion in face centered cubic co-cr-cu-fe-ni high-entropy alloys, Journal of Materials Science $\&$ Technology.

[18] Q. Li, W. Chen, J. Zhong, L. Zhang, Q. Chen, Z.-K. Liu, On sluggish diffusion in fcc alco-cr-fe-ni high-entropy alloys: An experimental and numerical study, Metals 8 (1) (2017) 16.

[19] C. Zhang, F. Zhang, K. Jin, H. Bei, S. Chen, W. Cao, J. Zhu, D. Lv, Understanding of the elemental diffusion behavior in concentrated solid solution alloys, Journal of Phase Equilibria and Diffusion 38 (4) (2017) 434-444.

[20] K.-Y. Tsai, M.-H. Tsai, J.-W. Yeh, Sluggish diffusion in co-cr-fe-mn-ni high-entropy alloys, Acta Materialia 61 (13) (2013) 4887 - 4897.

[21] A. Paul, Comments on "sluggish diffusion in co-cr-fe-mn-ni high-entropy alloys" by ky tsai, mh tsai and jw yeh, acta materialia 61 (2013) 4887-4897, Scripta Materialia 135 (2017) 153157.

[22] C. Lu, L. Niu, N. Chen, K. Jin, T. Yang, P. Xiu, Y. Zhang, F. Gao, H. Bei, S. Shi, M.-R. He, I. M. Robertson, W. J. Weber, L. Wang, Enhancing radiation tolerance by controlling defect mobility and migration pathways in multicomponent single-phase alloys, Nature Communications 7 (2016) 13564.

[23] T. Egami, W. Guo, P. D. Rack, T. Nagase, Irradiation Resistance of Multicomponent Alloys, Metallurgical and Materials Transactions A 45 (1) (2013) 180-183. 
[24] T. Nagase, S. Anada, P. D. Rack, J. H. Noh, H. Yasuda, H. Mori, T. Egami, MeV electronirradiation-induced structural change in the bcc phase of $\mathrm{Zr}-\mathrm{Hf}-\mathrm{Nb}$ alloy with an approximately equiatomic ratio, Intermetallics 38 (2013) 70-79.

[25] T. Nagase, S. Anada, P. D. Rack, J. H. Noh, H. Yasuda, H. Mori, T. Egami, Electronirradiation-induced structural change in Zr-Hf-Nb alloy, Intermetallics 26 (2012) 122-130.

[26] T. Nagase, P. D. Rack, J. H. Noh, T. Egami, In-situ TEM observation of structural changes in nano-crystalline $\mathrm{CoCrCuFeNi}$ multicomponent high-entropy alloy (HEA) under fast electron irradiation by high voltage electron microscopy (HVEM), Intermetallics 59 (2015) 32-42.

[27] Y. Zhang, M. A. Tunes, M. L. Crespillo, F. Zhang, W. L. Boldman, P. D. Rack, L. Jiang, X. Chen, G. Greaves, S. E. Donnelly, L. Wang, W. J. Weber, Thermal Stability and Irradiation Response of Nanocrystalline CoCrCuFeNi High-entropy Alloy (in press), Nanotechnology.

[28] F. Granberg, K. Nordlund, M. W. Ullah, K. Jin, C. Lu, H. Bei, L. M. Wang, F. Djurabekova, W. J. Weber, Y. Zhang, Mechanism of Radiation Damage Reduction in Equiatomic Multicomponent Single Phase Alloys, Physical Review Letters 116 (13) (2016) 1-8.

[29] N. K. Kumar, C. Li, K. Leonard, H. Bei, S. Zinkle, Microstructural stability and mechanical behavior of FeNiMnCr high entropy alloy under ion irradiation, Acta Materialia 113 (2016) 230-244.

[30] B. Kombaiah, P. Edmondson, Y. Wang, L. Boatner, Y. Zhang, Mechanisms of radiationinduced segregation around He bubbles in a Fe-Cr-Ni crystal, Journal of Nuclear Materials 514 (2019) 139-147.

[31] S. Zhao, Y. Osetsky, Y. Zhang, Preferential diffusion in concentrated solid solution alloys: NiFe, NiCo and NiCoCr, Acta Materialia 128 (2017) 391-399.

[32] K. Jin, C. Lu, L. Wang, J. Qu, W. Weber, Y. Zhang, H. Bei, Effects of compositional complexity on the ion-irradiation induced swelling and hardening in ni-containing equiatomic alloys, Scripta Materialia 119 (2016) 65-70.

[33] S. Zhao, W. J. Weber, Y. Zhang, Unique challenges for modeling defect dynamics in concentrated solid-solution alloys, JOM 69 (11) (2017) 2084-2091.

[34] S. Zhao, Y. Osetsky, G. M. Stocks, Y. Zhang, Local-environment dependence of stacking fault energies in concentrated solid-solution alloys, npj Computational Materials 5 (1) (2019) 13.

[35] L. K. Mansur, Void Swelling in Metals and Alloys Under Irradiation: An Assessment of the Theory, Nuclear Technology 40 (1) (1978) 5-34.

[36] S. E. Donnelly, The density and pressure of helium in bubbles in implanted metals: A critical review, Radiation Effects 90 (1-2) (2006) 1-47.

[37] J. Mayer, L. A. Giannuzzi, T. Kamino, J. Michael, Tem sample preparation and fib-induced damage, MRS bulletin 32 (5) (2007) 400-407.

[38] J. Trincavelli, S. Limandri, R. Bonetto, Standardless quantification methods in electron probe microanalysis, Spectrochimica Acta Part B: Atomic Spectroscopy 101 (2014) 76-85.

[39] J. Hinks, J. Van Den Berg, S. Donnelly, Miami: Microscope and ion accelerator for materials investigations, Journal of Vacuum Science \& Technology A: Vacuum, Surfaces, and Films 29 (2) (2011) 021003.

[40] C. A. Schneider, W. S. Rasband, K. W. Eliceiri, NIH Image to ImageJ: 25 years of image analysis, Nature Methods 9 (7) (2012) 671-675.

[41] M. Tunes, R. Harrison, G. Greaves, J. Hinks, S. Donnelly, Effect of He implantation on the microstructure of zircaloy-4 studied using in situ TEM, Journal of Nuclear Materials 493 (2017) 230-238. 
[42] R. W. Harrison, G. Greaves, J. Hinks, S. Donnelly, Engineering self-organising helium bubble lattices in tungsten, Scientific Reports 7.

[43] J. F. Ziegler, M. D. Ziegler, J. P. Biersack, Srim-the stopping and range of ions in matter (2010), Nuclear Instruments and Methods in Physics Research Section B: Beam Interactions with Materials and Atoms 268 (11) (2010) 1818-1823.

[44] R. E. Stoller, M. B. Toloczko, G. S. Was, A. G. Certain, S. Dwaraknath, F. A. Garner, On the use of SRIM for computing radiation damage exposure, Nuclear Instruments and Methods in Physics Research Section B: Beam Interactions with Materials and Atoms 310 (2013) 75-80.

[45] A. E521, Standard Practice for Neutron Radiation Damage Simulation by Charged-Particle, Annual Book of ASTM Standards 12.02 (Reapproved) (2009) 1-21.

[46] D. Rimmer, A. Cottrell, The solution of inert gas atoms in metals, Philosophical Magazine 2 (23) (1957) 1345-1353.

[47] H. Trinkaus, B. Singh, Helium accumulation in metals during irradiation - where do we stand?, Journal of Nuclear Materials 323 (2-3) (2003) 229-242.

[48] M. W. Thompson, Defects and radiation damage in metals, Defects and Radiation Damage in Metals, by MW Thompson, Cambridge, UK: Cambridge University Press, 19741.

[49] Y. Zhang, S. Zhao, W. J. Weber, K. Nordlund, F. Granberg, F. Djurabekova, Atomic-level heterogeneity and defect dynamics in concentrated solid-solution alloys, Current Opinion in Solid State and Materials Science 21 (5) (2017) 221-237.

[50] A. Calder, D. Bacon, A molecular dynamics study of displacement cascades in alpha-iron, Journal of Nuclear Materials 207 (1993) $25-45$.

[51] J. B. Gibson, A. N. Goland, M. Milgram, G. H. Vineyard, Dynamics of radiation damage, Phys. Rev. 120 (1960) 1229-1253.

[52] B. Wirth, G. Odette, J. Marian, L. Ventelon, J. Young-Vandersall, L. Zepeda-Ruiz, Multiscale modeling of radiation damage in fe-based alloys in the fusion environment, Journal of Nuclear Materials 329-333 (2004) 103 - 111, proceedings of the 11th International Conference on Fusion Reactor Materials (ICFRM-11).

[53] Y. N. Osetsky, L. K. Béland, A. V. Barashev, Y. Zhang, On the existence and origin of sluggish diffusion in chemically disordered concentrated alloys, Current Opinion in Solid State and Materials Science 22 (3) (2018) $65-74$.

[54] D. Terentyev, P. Olsson, T. Klaver, L. Malerba, On the migration and trapping of single selfinterstitial atoms in dilute and concentrated fe-cr alloys: Atomistic study and comparison with resistivity recovery experiments, Computational Materials Science 43 (4) (2008) 11831192.

[55] M. A. Tunes, F. C. da Silva, O. Camara, C. G. Schön, J. C. Sagás, L. C. Fontana, S. E. Donnelly, G. Greaves, P. D. Edmondson, Energetic particle irradiation study of tin coatings: are these films appropriate for accident tolerant fuels?, Journal of Nuclear Materials 512 (2018) 239-245.

[56] J. Winkelmann, Diffusion of xenon, in: Gases in Gases, Liquids and their Mixtures, Springer, 2007, pp. $152-155$.

[57] D. L. Morrison, T. S. Elleman, D. N. Sunderman, Diffusion of xenon in ceramic oxides, Journal of Applied Physics 35 (5) (1964) 1616-1622. 